\title{
Taxas de prenhez em novilhas de corte acasaladas aos 18 e 24 meses de idade
}

\author{
[Differences in pregnancy rate of beef heifers mating \\ at 18 and 24 months of age] \\ J.O.J. Barcellos ${ }^{1}$, M.D. Silva ${ }^{2}$, E.R. Prates ${ }^{1}$, E.C. Costa ${ }^{2}$ \\ ${ }^{1}$ Faculdade de Agronomia-UFRGS \\ Av. Bento Gonçalves, 7712 \\ 91540-000 - Porto Alegre \\ ${ }^{2}$ Aluno de pós-graduação - FA-UFRGS
}

\begin{abstract}
RESUMO
Avaliaram-se os efeitos da idade de acasalamento e classe de peso sobre a taxa de prenhez (TP) de novilhas de corte Hereford $(\mathrm{n}=119)$ acasaladas aos $18(18 \mathrm{M})$ ou 24 meses $(24 \mathrm{M})$ e classificadas em leves $(18 \mathrm{ML}=260 \mathrm{~kg} ; 24 \mathrm{ML}=280 \mathrm{~kg})$ e pesadas $(18 \mathrm{MP}=300 \mathrm{~kg} ; 24 \mathrm{MP}=330 \mathrm{~kg})$. O período de monta foi de $05 / 04$ a 21/05/01 (outono) e de $04 / 11$ a 20/12/01 (primavera) para $18 \mathrm{M}$ e $24 \mathrm{M}$, respectivamente. Avaliaram-se os efeitos do peso no início do acasalamento (PIA), escore da condição corporal (CCI) e ganho de peso (GDM). Não ocorreram diferenças ( $>>0,05)$ na TP entre os grupos 18MP $(73,3 \%), 24 \mathrm{ML}$ $(72,4 \%)$ e $24 \mathrm{MP}(83,3 \%)$, mas a TP foi mais baixa entre as novilhas $18 \mathrm{ML}(26,7 \%)$. O GDM foi maior para novilhas $24 \mathrm{M}(0,664 \mathrm{vs} 0,537 \mathrm{~kg} / \mathrm{d})(\mathrm{P}<0,05)$ que para as $18 \mathrm{M}$. No grupo $18 \mathrm{MP}$, as novilhas que conceberam tiveram maior GDM $(0,621$ vs $0,429 \mathrm{~kg} / \mathrm{d})$ em relação às falhadas. $\mathrm{O}$ CCI foi mais alto $(\mathrm{P}<0,10)$ nas novilhas que conceberam $(3,5)$ que nas falhadas $(3,32)$. Observou-se efeito quadrático do PIA $(\mathrm{P}<0,01)$ sobre a TP $\left(Y=-993,03+6,4543\right.$ PIA $\left.-0,0097 \mathrm{PIA}^{2} ; \mathrm{R}^{2}=0,98\right)$.
\end{abstract}

Palavras-chave: novilha, Hereford, acasalamento de outono, idade de acasalamento, peso vivo

\begin{abstract}
The effects of the mating age and weight class on the pregnancy rate (PR) of Hereford heifers $(n=119)$ at 18 (18M) or 24 months (24M) of age were evaluated. The heifers were divided into classes as light $(18 M L=260 \mathrm{~kg} ; 24 M L=280 \mathrm{~kg})$ or heavy $(18 M P=300 \mathrm{~kg} ; 24 M P=330 \mathrm{~kg})$. The breeding seasons were: 18M: 04/05-05/21 (fall) and 24M: 11/04-12/21 (spring). The traits analyzed were live weight (LW) and body condition score (BCS) at the beginning of the mating season and the average daily gain (ADG) on the pregnancy rate $(P R)$. The PR were $73.3 \%(18 M P), 72.4 \%(24 M L), 83.3 \%(24 M P)(P>0.05)$ and the $18 M L$ heifers have shown lower $P R(26.7 \%)(P<0.05)$. The $24 M$ heifers showed higher $A D G(0.664 v s$ $0.537 \mathrm{~kg} / \mathrm{d})$ than the $18 M$ heifers and in this group the open heifers had lower $A D G(0.429 \mathrm{vs} 0.621 \mathrm{~kg} / \mathrm{d})$ than the pregnant heifers. The BCS were higher $(P<0.10)$ for the pregnant heifers $(3.50)$ than the open (3.24) heifers. A quadratic effect of $L W$ on the PR $\left(Y=-993.03+6.4543 L W-0.0097 L W^{2} ; R^{2}=0.98\right)$ was observed.
\end{abstract}

Keywords: heifer, Hereford, fall mating, breeding age, live weight

Recebido em 10 de fevereiro de 2005

Aceito em 12 de junho de 2006

*Autor para correspondência (corresponding author)

E-mail: julio.barcellos@ufrgs.br 


\section{INTRODUÇ̃̃O}

$\mathrm{Na}$ produção de bovinos de corte, a eficiência da fase de cria é um dos fatores determinantes da viabilidade bioeconômica do sistema de produção. Segundo Gregory (1972) e Ferrel e Jenkins (1984), 65 a $75 \%$ da energia consumida no sistema é utilizada pelas matrizes destinadas à cria. Assim, nos sistemas de produção de bezerros, torna-se fundamental a diminuição de categorias de novilhas em recria, o que pode ser alcançado pela redução da idade de acasalamento, melhorando a eficiência global da produção, pois, as novilhas participam em proporção que varia de 15 a $20 \%$ do rebanho de matrizes, conforme a necessidade de reposição. Portanto, no primeiro acasalamento, a utilização de cerca de 10 a $15 \%$ de todo o recurso alimentar disponível para um sistema de ciclo completo depende da idade e do desempenho reprodutivo ao primeiro acasalamento.

A redução da idade de acasalamento, para reduzir a demanda nutricional da etapa da cria, requer uma antecipação do acasalamento de 24 para os 14 meses (Morrison et al., 1992; Potter et al., 1998). Contudo, esta diminuição pressupõe uma melhor recria das bezerras, o que em muitos casos inviabiliza economicamente o sistema (Freitas et al., 2003). Assim, etapas intermediárias, como o primeiro acasalamento aos 18 meses, na estação de outono, tem sido utilizada para melhorar a eficiência do sistema (Sampedro et al., 1995; Semmelman et al., 2001), sem incorrer num aumento substancial dos custos com alimentação
(Barcellos et al., 2003). Contudo, para viabilizar essa alternativa é fundamental que o desempenho reprodutivo dessas novilhas mantenha uma eficiência similar ao das acasaladas aos 24 meses. Além disso, torna-se estratégico conhecer os efeitos de parâmetros como o peso vivo ao acasalamento sobre a taxa de prenhez, cuja relação é bem documentada em novilhas acasaladas aos 14 ou 24 meses (Chapman et al., 1978; Cohen et al., 1980). Entretanto, em se tratando de novilhas acasaladas aos 18 meses poucas informações são disponíveis. O objetivo do experimento foi avaliar os efeitos da idade e do peso vivo ao início do acasalamento sobre a taxa de prenhez de novilhas de corte.

\section{MATERIAL E MÉTODOS}

Utilizaram-se 119 novilhas Hereford, nascidas na primavera de 1999 e mantidas no mesmo sistema de recria até o início do experimento, com 18 meses de idade, distribuídas em quatro grupos experimentais em 5 de abril de 2001 (Tab.1), conforme o peso, a condição corporal e a data de nascimento. Dois grupos, denominados leve (18ML) e pesado (18MP) foram escolhidos para acasalamento aos 18 meses de idade, o qual ocorreu de 05/04 a 21/05/2001, no outono. Os outros dois, denominados leve (24ML) e pesado (24MP) foram escolhidos para acasalamento aos 24 meses de idade, ocorrido na primavera/verão, de $04 / 11$ a $20 / 12 / 2001$.

Tabela 1. Distribuição das novilhas de corte conforme o tratamento, idade de acasalamento, estação reprodutiva e peso vivo no início do acasalamento

\begin{tabular}{ccccc}
\hline Tratamentos & $\mathrm{n}$ & $\begin{array}{c}\text { Idade ao } \\
\text { acasalamento }\end{array}$ & Estação de acasalamento & $\begin{array}{c}\text { Peso ao início do } \\
\text { acasalamento }\end{array}$ \\
\hline $18 \mathrm{MP}$ & 30 & 18 meses & $05 / 04-21 / 05 / 2001$ & $301 \mathrm{~kg}$ \\
$18 \mathrm{ML}$ & 30 & 18 meses & $05 / 04-21 / 05 / 2001$ & $264 \mathrm{~kg}$ \\
$24 \mathrm{MP}$ & 29 & 24 meses & $04 / 11-20 / 12 / 2001$ & $331 \mathrm{~kg}$ \\
$24 \mathrm{ML}$ & 30 & 24 meses & $04 / 11-20 / 12 / 2001$ & $285 \mathrm{~kg}$ \\
\hline
\end{tabular}

$18 \mathrm{MP}=$ novilhas com 18 meses (pesadas); $18 \mathrm{ML}=8$ meses (leves); $24 \mathrm{MP}=24$ meses (pesadas); 24 $\mathrm{ML}=24$ meses (leves).

O acasalamento foi o de monta natural, utilizando-se dois touros da raça Hereford em cada grupo experimental, em piquetes de campo nativo, com carga animal média de $300 \mathrm{~kg} / \mathrm{ha}$. Nesses piquetes foram mantidas disponibilidades de forragem em torno de $2800 \mathrm{kgMS} /$ ha. Após o acasalamento de outono até o acasalamento de primavera, as novilhas permaneceram em um mesmo piquete, quando então as novilhas do tratamento $24 \mathrm{M}$ foram acasaladas.

A taxa de prenhez (TP) foi obtida pelo diagnóstico de gestação, através de palpação retal realizado 90 dias do término das temporadas de acasalamento. 
As pesagens foram realizadas no início da manhã, após período de jejum de 12 horas, quando também foi realizada a avaliação subjetiva da condição corporal conforme adaptação da escala de Lowman et al. (1973), com escores de 1 a 5 , onde o escore 1 representa um animal muito magro e o escore $5 \mathrm{um}$ animal muito gordo. A partir dessas medidas foram obtidos o peso no início (PIA) do acasalamento, ganho diário de peso (GDM) durante o acasalamento e o escore de condição corporal (CCI) no início do acasalamento.

Durante as temporadas de acasalamento foram coletadas amostras de pasto, por meio do método descrito por t'Mannetje (1978), para medir a disponibilidade e a qualidade da forragem. As amostras foram secas em estufa de ar forçado em temperatura de $60^{\circ} \mathrm{C}$ durante três dias para determinação dos teores de matéria seca (MS), proteína bruta $(\mathrm{PB})$, fibra em detergente neutro (FDN) e fibra em detergente ácido (FDA).

Usou-se delineamento inteiramente ao acaso, com arranjo fatorial $2 \times 2$ (idade de acasalamento e categoria de peso), com número desigual de repetições. Para análise dos dados usou-se o programa estatístico SPSS (User's...,2002). Para as respostas do PIA e GDM usou-se o teste Tukey para testar as diferenças entre médias ajustadas.

$Y_{i j k}=\mu+T_{i}+E_{j}+T_{i j}+\varepsilon_{i j k}$, em que:

$\mathrm{Y}_{\mathrm{ijk}}=$ é a observação do indivíduo $\mathrm{k}$, na idade de acasalamento $\mathrm{i}$, na categoria de peso $\mathrm{j}$,

$\mu=$ média geral,

$\mathrm{T}_{\mathrm{i}}=$ é o efeito da idade de acasalamento $\mathrm{i}$,

$E_{j}=$ é o efeito da categoria de peso $\mathrm{j}$,

$\mathrm{TE}_{\mathrm{ij}}=$ é o efeito da interação idade de acasalamento $\mathrm{x}$ categoria de peso,

$\varepsilon_{\mathrm{ijk}}=$ é $\mathrm{o}$ erro aleatório inerente a cada observação.

Para comparação dos dados do escore de condição corporal (CCI) no início do acasalamento usou-se método não paramétrico de Kruskal-Wallis (User's..., 2002), e para os dados referentes à taxa de prenhez usou-se o teste do qui-quadrado $\left(\chi^{2}\right)$.

\section{RESULTADOS E DISCUSSÃO}

Na Tab. 2 são apresentadas as TP segundo a idade e a classe de peso no início do primeiro acasalamento.

Tabela 2. Taxa de prenhez (\%) de novilhas de corte segundo a idade e a classe de peso no início do acasalamento

\begin{tabular}{ccc}
\hline Grupo experimental & Leve (L) & Pesada (P) \\
\hline $18 \mathrm{M}$ & $26,7 \mathrm{aA}$ & $73,3 \mathrm{bA}$ \\
$24 \mathrm{M}$ & $72,4 \mathrm{aB}$ & $83,3 \mathrm{aA}$ \\
\hline
\end{tabular}

$\mathrm{M}=$ meses. Médias seguidas de letras minúsculas distintas na linha ou maiúsculas distintas na coluna diferem entre si $(\mathrm{P}<0,05)$.

A TP foi influenciada pela idade e pelo PIA $(\mathrm{P}<0,05)$, e o efeito da interação entre as duas variáveis foi significativo. As novilhas acasaladas aos 24 meses de idade, na primavera, apenas apresentaram maior TP que as novilhas leves (L) acasaladas aos 18 meses $(\mathrm{P}<0,05)$. Essa diferença pode ser atribuída ao efeito do PIA nos animais acasalados em idades mais jovens.

No grupo $18 \mathrm{ML}$, o peso no início da estação reprodutiva representava $55 \%$ do peso da vaca adulta no mesmo rebanho $(480 \mathrm{~kg})$, o que pode explicar a menor taxa de prenhez em relação aos outros grupos experimentais, aspectos já observados por Cohen et al. (1980); Nicoll (1990) e Spire (1997). Sampedro et al. (1995), ao analisarem grande número de dados de novilhas acasaladas aos 18 meses, em regiões subtropicais, verificaram alta correlação entre o peso no início do outono e o índice de concepção. Semmelmann et al. (2001) obtiveram em novilhas Nelore com 18 meses de idade e peso de $262 \mathrm{~kg}$, um índice de prenhez de $20 \%$, semelhante ao alcançado pelas novilhas do grupo $18 \mathrm{ML}$.

Silva et al. (2005) observaram taxa de prenhez de $52,2 \%$ em novilhas Hereford, acasaladas aos 18 meses de idade, e com peso ao início da estação reprodutiva de $286 \mathrm{~kg}$, valores intermediários entre as novilhas dos grupos $18 \mathrm{ML}$ e $18 \mathrm{MP}$ observados neste estudo.

Nardon (1985), ao avaliar o desempenho reprodutivo de novilhas acasaladas aos 24 meses de idade, com peso médio aos dois anos, semelhante aos encontrados nos grupos denominados leves do presente trabalho, observou taxa de prenhez média 
de 58,5\%. Wolfe et al. (1995) encontraram índices de prenhez em novilhas Hereford com pesos iniciais de 286 a $323 \mathrm{~kg}$ que variavam de 77 a $90 \%$, respectivamente.

A análise entre os pesos das novilhas que conceberam e das que falharam (Tab. 3) mostram a relevância do PIA sobre a eficiência reprodutiva. Aos 24 meses, as novilhas que conceberam, independentemente da classe de peso, eram mais pesadas $(\mathrm{P}<0,05)$ no início do acasalamento que as falhadas. Aos $18 \mathrm{M}$ ocorreu interação $(\mathrm{P}<0,05)$ entre PIA e classe de peso das novilhas para a resposta prenhe ou falhada. Somente no $18 \mathrm{ML}$, as novilhas que conceberam foram mais pesadas que as que falharam $(\mathrm{P}<0,05)$. Isso sugere que aos 18 meses, após alcançar um peso mínimo, provavelmente nas novilhas do grupo 18MP, o PIA exerceu menor efeito sobre a probabilidade de prenhez. Desse modo, a taxa de prenhez pode estar sendo influenciada por outros fatores, como o ganho de peso durante o acasalamento. Alguns autores (Wiltbank et al., 1985; Pereira Neto e Lobato, 1998; Semmelmann et al., 2001) encontraram diferenças de outras magnitudes entre prenhes e vazias, geralmente associadas a outros fatores não diretamente ligados ao peso no acasalamento.

Tabela 3. Peso no início do acasalamento (PIA) de novilhas prenhes e falhadas

\begin{tabular}{|c|c|c|}
\hline \multirow{2}{*}{ Grupo experimental } & \multicolumn{2}{|c|}{ PIA (kg) } \\
\hline & Prenhes & Não prenhes \\
\hline $18 \mathrm{ML}$ & $273,4 a$ & $261,0 \mathrm{~b}$ \\
\hline $18 \mathrm{MP}$ & $302,1 \mathrm{a}$ & $298,2 \mathrm{a}$ \\
\hline $24 \mathrm{ML}$ & $289,2 \mathrm{a}$ & $271,4 b$ \\
\hline $24 \mathrm{MP}$ & $334,5 \mathrm{a}$ & $315,6 b$ \\
\hline
\end{tabular}

Médias seguidas de letras distintas na linha diferem entre si $(\mathrm{P}<0,05)$.

Essa análise dos pesos entre novilhas que conceberam ou não é uma maneira de mostrar a importância de pesos mais altos ao início da temporada de acasalamento para que sejam alcançados índices de prenhez compatíveis com o aumento de produtividade e, por conseqüência, melhor eficiência do sistema produtivo.

Verificou-se efeito da idade $(\mathrm{P}<0,05)$ sobre o GDM, isto é, as novilhas do grupo $24 \mathrm{M}$ apresentaram maior GDM do que as do grupo $18 \mathrm{M}$ $(0,664$ vs $0,537 \mathrm{~kg} / \mathrm{d})$.

Os dados do grupo $18 \mathrm{M}$ são semelhantes aos obtidos por outros autores que realizaram experimentos avaliando desempenhos reprodutivos de novilhas acasaladas no outono com carga animal de $320 \mathrm{~kg} /$ hectare (Barcellos et al., 2000; Montanholi et al., 2003).

A diferença de GDM entre idades, provavelmente, resulta de diferenças metabólicas atribuídas ao estágio de desenvolvimento conforme citado pelo NRC (Nutrient..., 1996), associadas às variações na qualidade da forragem, visto que o pastejo ocorreu em épocas distintas, isto é, as novilhas do 24M pastejaram forragens com maior teor de PB $(9,1 \mathrm{vs}$ $7,4 \%)$ e menor de FDN (71,2 vs $81,0 \%)$ que as do grupo $18 \mathrm{M}$.
Embora as diferenças no GDM sejam de apenas $0,127 \mathrm{~kg} / \mathrm{d}$ entre os dois grupos de idade, elas podem ter influenciado o desempenho reprodutivo. Foram observadas diferenças no GDM entre novilhas que conceberam ou que falharam apenas no grupo $18 \mathrm{MP}(0,621$ vs $0,429 \mathrm{~kg} / \mathrm{d})$, o que pode explicar o fato de as novilhas desse grupo não terem apresentado diferenças nos PIA entre prenhes e falhadas $(302,1$ vs $298,2 \mathrm{~kg})$. Portanto, como o grupo $18 \mathrm{M}$ apresentou menor GDM, sua contribuição para que as novilhas alcançassem o peso mínimo para concepção foi pequeno, especialmente nas novilhas leves. Nas novilhas pesadas, que já apresentavam peso mínimo favorável à concepção, o efeito do GDM foi independente, o que demonstra a importância dessa variável durante o acasalamento para o sucesso reprodutivo, especialmente numa temporada reprodutiva de curta duração, como foi o caso deste experimento.

Não foram identificados efeitos $(\mathrm{P}>0,10)$ da idade $\mathrm{e}$ da classe de peso das novilhas sobre o escore de condição corporal no início do acasalamento. Essa é uma característica de menor relevância na avaliação do desenvolvimento de novilhas jovens, pois em animais em crescimento as diferenças existentes são de difícil visualização, o que explica, muitas vezes, a ausência de diferenças no escore de condição corporal entre tratamentos (Brooks et al., 1985; Beretta e Lobato, 1998; Vargas et al., 1999). 
As novilhas prenhes apresentam-se com maior CCI $(3,50)$ que as falhadas $(3,24)$ no final do período reprodutivo $(\mathrm{P}<0,10 \%)$.

Os dados deste experimento evidenciam que, uma vez alcançado o peso mínimo ao acasalamento, o CCI passa a exercer efeito positivo sobre a probabilidade de prenhez da novilha, sendo mais importante nas novilhas acasaladas aos 18 meses de idade. Quando elas não alcançam um peso mínimo, provavelmente o caso das novilhas dos grupos $18 \mathrm{ML}$ e $24 \mathrm{ML}$, esse efeito não é demonstrado, fato já descrito por Semmelmann et al., (2001).
É importante também ressaltar que tanto o PIA quanto o CCI foram avaliações pontuais, na ocasião do início das temporadas de acasalamento. A partir dessas datas, ocorreram modificações no estado nutricional dos animais, resultante das variações de peso observadas durante o período de 46 dias de acasalamento.

A regressão múltipla, incluindo no modelo PIA, GDM, CCI e idade da novilha, na tentativa de avaliar a contribuição de cada característica sobre a taxa de prenhez, mostrou efeito significativo $(\mathrm{P}<0,01)$ apenas do PIA, sendo desse modo, as demais variáveis excluídas do modelo inicial (Fig. 1).

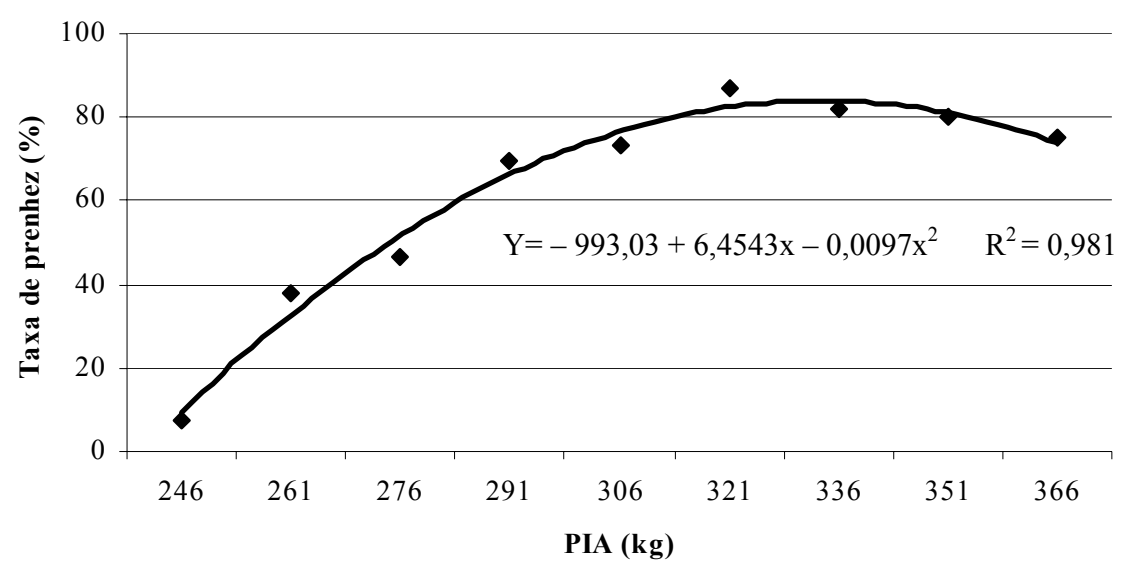

Figura 1. Regressão do peso no início do acasalamento (PIA) sobre a taxa de prenhez.

Nas novilhas com maior PIA (246 e 332kg) observou-se maior probabilidade de prenhez. Entretanto, a partir desse ponto de máximo, ocorreu inflexão na curva, o que evidencia que após atingir esse peso, o aumento de peso não implica maior probabilidade de prenhez. Deste modo, é factível concluir que outros fatores possam estar influenciando a reprodução. Cohen et al. (1980), ao analisarem aspectos reprodutivos de novilhas Hereford, verificaram manifestação de cio de $5 \%$ e $95 \%$ em animais com pesos de $187 \mathrm{~kg}$ e $280 \mathrm{~kg}$, respectivamente. Esses autores observaram que nas novilhas com $231 \mathrm{~kg}$, metade delas alcançaram a puberdade. Segundo Ellis (1974), a relação entre peso ao início do acasalamento e taxa de parição é linear dos $175 \mathrm{~kg}$ aos $265 \mathrm{~kg}$. Nessa amplitude, para cada $10 \mathrm{~kg}$ de incremento no peso, a taxa de parição aumentou 7\%. Com base nos resultados é possível sugerir que a variável PIA tenha fundamental importância na busca de melhores índices reprodutivos.

\section{CONCLUSÕES}

O peso no início do acasalamento é a variável de maior impacto sobre a eficiência reprodutiva durante o primeiro acasalamento de novilhas de corte. Novilhas cobertas aos 18 meses, leves, necessitam de maior ganho de peso no período de acasalamento para atingir taxas de prenhez semelhantes às de novilhas acasaladas aos 24 meses.

\section{REFERÊNCIAS BIBLIOGRÁFICAS}

BARCELlOS, J.O.J.; COSTA, E.C.; SILVA, M.D. et al. Crescimento de fêmeas bovinas de corte aplicado aos sistemas de cria. Porto Alegre: UFRS/Faculdade de Agronomia. 2003. 72p. 
BARCELLOS, J.O.J.; PATIÑO, H.O.; PRATES, E.R. et al. Carga animal pós-desmama e desempenho reprodutivo de novilhas de corte acasaladas aos 18 meses de idade. In: REUNIÃO ANUAL DA SOCIEDADE BRASILEIRA DE ZOOTECNIA, 37., 2000, Viçosa. Anais ..., Viçosa: SBZ, 2000. CD-ROM.

BERETTA, V.; LOBATO, J.F.P. Sistema "um ano" de produção de carne: avaliação de estratégias alternativas de alimentação hibernal de novilhas de reposição. Rev. Bras. Zootec., v.27, p.157-163, 1998.

BROOKS, A.L.; MORROW, R.E.; YOUNGQUIST, R.S. Body composition of beef heifers at puberty. Theriogenology, v.24, p.235-250, 1985.

COHEN, R.D.H.; GARDNER, D.L.; LANGLANDS, J.P. A note on the relationship between live weight and incidence of oestrous in Hereford heifers. Anim. Prod., v.31, p.221-222, 1980.

CHAPMAN, H.D.; YOUNG, J.M.; MORRISON, E.G. et al. Differences in lifetime productivity of Hereford calving first at 2 and 3 years of age. J. Anim. Sci., v.46, p.1159-1162, 1978.

ELLIS, R.W. The relationship between percentage calving and weight at joining in early Hereford heifers. Proc. Austr. Soc. Anim. Prod., v.10, p.55-58, 1974.

FERREL, C.L.; JENKINS, T. G. Energy utilization by mature, nonpregnant, nonlactating cows of different types. J. Anim. Sci., v.58, p.234-243, 1984.

FREITAS, S.G.; LOBATO, J.F.P.; TAROUCO, A. et al. Desempenho reprodutivo e produtivo de novilhas de corte aos dois anos de idade submetidas a diferentes alternativas de alimentação. In: REUNIÃO ANUAL DA SOCIEDADE BRASILEIRA DE ZOOTECNIA, 40., 2003, Santa Maria. Anais..., Santa Maria: SBZ, 2003. CD-ROM.

GREGORY, K.E. Beef cattle type for maximum efficiency "putting it all together". J. Anim. Sci., v.34, p.881-884, 1972.

LOWMAN, B.G.; SCOTT, N.; SOMERVILLE, S. Condition scoring beef cattle. Edinburgh: East of Scotland College of Agriculture, 1973. 8p. (Bulletin, 6).

MONTANHOLI, Y.R.; BARCELLOS, J.O.J.; COSTA, E.C. et al. Effects of stocking rate on the yearling beef heifers. In: WORLD CONFERENCE ON ANIMAL PRODUCTION, 9., 2003, Porto Alegre. Proceedings..., Porto Alegre: WAAP, 2003. CDROM.

MORRISON, D. G.; FEAZEL, J. I.; BAGLEY, C. P. et al. Postweaning growth and reproduction of beef heifers exposed to calve at 24 or 30 months of age in spring and fall seasons. J. Anim. Sci., v.70, p.622-630, 2002.
NARDON, R.F. Desenvolvimento e comportamento de fêmeas de corte em pastagens. 1985. 144f. Dissertação (Mestrado) - Universidade Federal do Rio Grande do Sul, Faculdade de Agronomia, Porto Alegre.

NICOLL, A.M. Beef cattle production. Lincoln: Canterbury University College of Agriculture, 1990. 100p.

NUTRIENT requirements of cattle. Washington DC: National Academy, 1996. 242p.

PEREIRA NETO, O.A.; LOBATO, J.F.P. Efeitos da ordem de utilização de pastagens nativas melhoradas no desenvolvimento e comportamento reprodutivo de novilhas de corte. Rev. Bras. Zootec., v.27, p.60-65, 1998.

POTTER, L.; LOBATO, J.F.P.; NETTO, C.G.M., Produtividade de um modelo de produção para novilhas de corte primíparas aos dois, três ou quatro anos de idade. Rev. Bras. Zootec., v.27, p.613-619, 1998.

SAMPEDRO, D.; VOGEL, O.; CELSER, R. Alternativas de manejo para entorar la vaquilla a los 18 meses de edad: su influencia sobre el porcentaje de $2^{\circ}$ entore y prenhez. Mercedes: INTA, 1995. 9p. (Circular Técnica).

SEMMELMANN, C.E.N.; LOBATO, J.F.P.; ROCHA, M.G. Efeito de sistemas de alimentação no ganho de peso e desempenho reprodutivo de novilhas Nelore acasaladas aos 17-18 meses. Rev. Bras. Zootec., v.30, p.835-843, 2001.

SILVA, M.D.; BARCELLOS, J.O.J.; PRATES, E.R. Desempenho reprodutivo de novilhas de corte acasaladas aos 18 ou aos 24 meses de idade. Rev. Bras. Zootec., v.34, p.2057-2063, 2005.

SPIRE, M.F. Managing replacement heifers from weaning to breeding. Vet. Med., v. 92, p.182-192, 1997.

t MANNETJE, L. Measuring quantity grassland vegetation. In: 't MANNETJE, L. Measurement of grassland vegetation and animal production. London: Farnham Royal : Commonwealth Agricultural Bureaux, 1978. p.36-90, (Bulletin, 52).

USER'S guide: statistics. SPSS Inc Version 11.5. Chicago. IL: Headquarters, 2002.

VARGAS, C.A.; OLSON, T.A.; CHASE, C.C. et al. Influence of frame size and body condition score on performance of Brahman cattle. J. Anim. Sci., v.77, p.3140-3149, 1999.

WILTBANK, J.N.; ROBERTS, S.; NIX, J. et al. Reproductive performance and profitability of heifers fed to weight 272 or $318 \mathrm{~kg}$ at the start of the breeding season. J. Anim. Sci., v.60, p.25-34, 1985. 


\section{Barcellos et al.}

WOLFE, M.W.; STUMPF, T.T.; WOLFE, P.L. et al. Effect of selection for growth traits on age and weight at puberty in bovine females. J. Anim. Sci., v.68, p.1595-1602, 1990. 